\begin{tabular}{|c|l|}
\hline Title & Ionization and excitation collision processes of electrons in liquid water \\
\hline Author(s) & Date, H.; Sutherland, K. L.; Hasegawa, H.; Shimcizurna, M. \\
\hline Citation & $\begin{array}{l}\text { Nuclear Instruments and Methods in Physics Research Section B: Beam Interactions with Materials and A toms, 265(2), } \\
515-520 \\
\text { https://doi.org/L0.1016/.nimb.2007.09.039 }\end{array}$ \\
\hline Issue Date & $2007-12$ \\
\hline Doc URL & http://hdl.handle.net/2115/32337 \\
\hline Type & article (author version) \\
\hline File Information & Date_NIMB_HUSCAP_Feb.2008.pdf \\
\hline
\end{tabular}

Instructions for use 


\title{
Ionization and Excitation Collision Processes of Electrons in Liquid Water
}

\author{
H. Date*, K.L. Sutherland ${ }^{1}$, H. Hasegawa ${ }^{2}$, M. Shimozuma ${ }^{3}$ \\ School of Medicine, Hokkaido University, Sapporo 060-0812, Japan \\ ${ }^{1}$ Japan Science and Technology Agency, Tokyo 105-6218, Japan \\ ${ }^{2}$ Tomakomai National College of Technology, Tomakomai 059-1275, Japan \\ ${ }^{3}$ Hokkaido Institute of Technology, Sapporo 006-8585, Japan
}

\begin{abstract}
This paper reports on Monte Carlo simulations of electrons in liquid water using a set of electron collision cross sections constructed with data published recently. The track history of electrons having initial energy ranged from $1 \mathrm{keV}$ to $10 \mathrm{keV}$ is investigated looking at the ionization and excitation processes. The results show that the ratio of the ionization and excitation events per track history is unique independent of the initial electron energy above a couple of hundred $\mathrm{eV}$ and these inelastic processes occur with low energy electrons frequently below $100 \mathrm{eV}$. In particular, the excitation processes are dominated by the electrons below 50eV. Flight distance distributions between the inelastic collisions are also discussed.
\end{abstract}

\section{Keywords}

Electron collision cross sections, Inelastic collisions, Ionization, Excitation, Liquid water

PACS numbers: $34.80 . \mathrm{Dp}$

*Corresponding author: H. Date

Postal address: N12-W5, Kita-ku, Sapporo 060-0812, JAPAN

Email address: date@cme.hokudai.ac.jp

Tel: +81-11-706-3423, Fax: +81-11-706-4916 


\section{Introduction}

In irradiated bio-tissues, the final entities to interact with the material molecules producing excited species and ions are mostly electrons that have taken over energy from the primary incident particles. The electrons undergo stochastic collision processes transferring their energy to the molecules, which may cause indirect or direct damage to the structures in bio-cells.

Within this framework, we have been performing Monte Carlo simulations of electrons in water as a principal component of biological material. The most essential parameter affecting the results such as inelastic collision frequencies per track history is the electron collision cross section of the water molecule. We have constructed a set of cross sections for elastic, excitation and ionization processes in both vapor phase and liquid phase water from published data including recently reported, and investigated the characteristics of electron tracks.

This paper reports an investigation using the Monte Carlo simulation technique on the ionization and excitation processes by electrons below several keVs energy in liquid water. By taking account of thousands of track histories, we have deduced general features of electron processes. In spite of high energies above $1 \mathrm{keV}$ of the incident electrons, average energy of progeny electrons produced in the track history is quite low. Our calculation results show that a great number of electrons below $100 \mathrm{eV}$ are essential to ionize and excite the water molecules.

The present work will be of use to estimate the ionization and excitation events occurring in bio-cells, which may contribute to the study of nano-dosimetry.

\section{Electron collision cross sections in liquid water}

The method of Monte Carlo simulation is based upon the determination of flight distance between two successive collisions given by 


$$
\ell=-\frac{\ln \xi}{\sigma_{T} N}
$$

where $\sigma_{\mathrm{T}}$ is the total collision cross section, $\mathrm{N}$ is the number density of target particles and $\xi$ is a random number. In our simulation, the total collision cross section consists of elastic, total electronic excitation, vibrational excitation, total ionization and attachment cross sections. Here, "total ionization" means the sum of ionization processes producing $\mathrm{OH}^{+}, \mathrm{O}^{+}$and so on including $\mathrm{H}_{2} \mathrm{O}^{+}$. For vapor phase water, the electronic excitation and ionization cross sections can be divided into lots of cross sections of various excitation levels and ionization species since there are numerous data reported to give each cross section (e.g. [1][2]). However, the cross section data for liquid phase water are limited and at this moment only the total electronic excitation and ionization cross sections are available. Recently, Emfietzoglou et al $[3,4,5]$ presented both of the cross sections in liquid water in quick succession, which were determined by the optical method using a dielectric response model. Although their cross sections may still have uncertainty as stated in their paper [4] and room for further considerations, the general feature for the excitation and ionization cross sections seems acceptable from a consistency with other reported data [6][7][8]. For instance, the ionization cross section is much smaller in low energy below $100 \mathrm{eV}$ than that of vapor phase water molecule while the excitation cross section is smaller in the entire energy region below several tens of $\mathrm{keV}$. Therefore, we have decided to utilize the excitation and ionization cross sections by them for our simulation, respectively as the total electronic excitation and total ionization cross sections in liquid water. The threshold values for the ionization and the electronic excitation are $13.65 \mathrm{eV}$ and $8.22 \mathrm{eV}$, respectively.

In addition to the electronic excitation and the ionization of water molecules, we also consider the vibrational excitation and electron attachment processes because the cross sections for them are overlying the electronic excitation and ionization cross sections around their threshold energies. These processes are important at the endpoint of the track simulation 
with low energies decreased from high energy regions of electron because the number of final products from water molecules varies depending on the processes. In our simulations, three cross sections for the vibrational excitation and three attachment cross sections are taken into account, and those are assumed to be the same as water vapor. As the vibration excitation cross sections $\mathrm{q}_{\mathrm{vib}}$, three lump modes, $(100+001),(010)$ and others, are considered. The cross sections for $(100+001)$ and $(010)$ are constructed by a combination of the data from Seng and Linder (1976) [9] and El-Zein et al (2000) [10] where the cross section around 0.3-6eV is taken from Seng and Linder, and for $6-20 \mathrm{eV}$ is from El-Zein et al. The summed cross section for the other modes is estimated referring to Märk et al (1995) [11]. The electron attachment cross sections for the production of $\mathrm{OH}^{-}, \mathrm{O}^{-}$and $\mathrm{H}^{-}$are taken from Melton (1972) [12].

As to the elastic cross section, we have adopted the cross section by Danjo and Nishimura [13] with multiplication factor of 1.4 so as to agree with the total collision cross section by Itikawa and Mason (2005) [14]. This is because the elastic cross section for liquid water has scarcely been reported to our knowledge. However, note that the elastic collision process does not contribute so much to the energy transfer from electrons to targets. Because the cutoff energy for chasing an electron track history is set to be the electronic excitation threshold (i.e., $8.22 \mathrm{eV})$, the low energy part of the elastic cross section below the threshold is not necessary and therefore we assume it is constant as a dummy in our simulation.

The set of electron collision cross sections for liquid water used in the simulation is shown in Fig.1.

\section{Simulation algorithm}

While the flight distance between two successive collisions is given by Eq.(1) mentioned above, the collision type (i.e., elastic, excitation and ionization, etc.) and the direction of electron after the collision are also determined using random numbers. Although the basic algorithms in the Monte Carlo method are similar to those published so far [16], briefly we 
describe them below.

For the determination of the direction of scattered electron after the elastic collision, we use semi-empirical equations by Brenner and Zaider (1983) [15]. They presented the equations for scattering probabilities toward the polar angle $\omega$ with respect to the incident electron as follows:

$$
\begin{array}{ll}
\left(\frac{\mathrm{d} \sigma}{\mathrm{d} \Omega}\right) \propto \frac{1}{(1+2 \eta-\cos \omega)^{2}} & \text { for } 200 \mathrm{eV}<\mathrm{E} \\
\left(\frac{\mathrm{d} \sigma}{\mathrm{d} \Omega}\right) \propto \frac{1}{(1+2 \gamma-\cos \omega)^{2}}+\frac{\alpha}{(1+2 \delta+\cos \omega)^{2}} & \text { for } 0.35 \mathrm{eV} \leq \mathrm{E} \leq 200 \mathrm{eV}
\end{array}
$$

Here, $E$ is the incident electron energy, $\mathrm{d} \sigma / \mathrm{d} \Omega$ is the differential cross section, $\eta, \alpha$ and $\delta$ are parameters. These parameters are given in their paper to fit experimental angular distribution for elastic collision. Thus the scattering angle $\omega$ can be determined with a random number छ' satisfying

$$
\xi^{\prime}=\frac{2 \pi \int_{0}^{\omega}\left(\frac{d \sigma}{d \Omega}\right) \sin \omega \mathrm{d} \omega}{2 \pi \int_{0}^{\pi}\left(\frac{d \sigma}{d \Omega}\right) \sin \omega \mathrm{d} \omega} .
$$

An important factor in handling the ionization process is the energy partition to two electrons after the ionization collision, where the residual energy of an incident electron, E-I, is divided into primary and secondary electrons. Here, E is kinetic energy of the incident electron and I the ionization threshold energy. The electron that has a higher energy is called "primary" and the other is "secondary". For selecting the energy partition ratio of both electrons, we use an algorithm by Grosswendt and Waibel (1978) [16]. In their algorithm, the energy of the secondary electrons is determined by

$$
\mathrm{T}=\mathrm{T}_{0}+\Gamma \tan \left\{\xi^{\prime \prime}\left[\tan ^{-1}\left(\frac{\mathrm{T}_{\mathrm{m}}-\mathrm{T}_{0}}{\Gamma}\right)+\tan ^{-1}\left(\frac{\mathrm{T}_{0}}{\Gamma}\right)\right]-\tan ^{-1}\left(\frac{\mathrm{T}_{0}}{\Gamma}\right)\right\} .
$$

Here, $\mathrm{T}_{0}=\mathrm{T}_{\mathrm{s}}-\mathrm{T}_{\mathrm{a}} /\left(\mathrm{E}+\mathrm{T}_{\mathrm{b}}\right), \mathrm{T}_{\mathrm{m}}=(\mathrm{E}-\mathrm{I}) / 2, \Gamma=\Gamma_{\mathrm{s}} \mathrm{E} /\left(\mathrm{E}+\Gamma_{\mathrm{b}}\right)$ and $\zeta$ " is a random number, $\mathrm{T}_{\mathrm{s}}, \mathrm{T}_{\mathrm{a}}, \mathrm{T}_{\mathrm{b}}, \Gamma_{\mathrm{s}}$ and $\Gamma_{\mathrm{b}}$ are fitting parameters taken from publication of Green and Sawada (1972) [17] for 
vapor phase water. Although the use of the parameters for gas phase water is problematic, we employ these with a slight modification according to the ionization threshold value since the energy distribution of secondary electrons for liquid water has been hardly measured. At the same time, the directions of the primary and secondary electrons are determined from the kinematic relationships,

$$
\sin ^{2} \theta_{p}=\frac{T / E}{(1-T / E) \cdot \frac{E}{2 m_{0} c^{2}}+1}=\frac{2 m_{0} c^{2}}{(E-T)+2 m_{0} c^{2}} \cdot \frac{T}{E}
$$

for the primary electron and

$$
\sin ^{2} \theta_{\mathrm{s}}=\frac{1-\mathrm{T} / \mathrm{E}}{1+\frac{\mathrm{T}}{2 \mathrm{~m}_{0} \mathrm{c}^{2}}}
$$

for the secondary electron. Here, $\theta_{\mathrm{p}}$ and $\theta_{\mathrm{s}}$ are the polar angles with respect to the direction of the incident electron for the primary and the secondary electrons, respectively. The azimuthal angle $\phi_{\mathrm{p}}$ of the primary electron is uniformly distributed between 0 and $2 \pi$, and the azimuthal angle $\phi_{\mathrm{s}}$ for the secondary electron is set equal to $\phi_{\mathrm{p}}-\pi$.

The electron after the excitation collision is assumed to go out in the same direction as the incident electron with a residual energy, $\mathrm{E}-\mathrm{I}_{\mathrm{exc}}$, where $\mathrm{I}_{\mathrm{exc}}$ is the excitation threshold energy.

\section{Results and discussion}

By using the procedure mentioned above, we have performed Monte Carlo simulations of the track of electrons incident on liquid water. The trial number of the incident electrons is typically $1000-5000$ in the present study. The initial electron energy was set to be from $1 \mathrm{keV}$ to $10 \mathrm{keV}$. This is because radiation particles typically used in medical fields, e.g., X-rays and protons, produce abundant electrons with energies much lower than those of the primary particles in bio-tissues. For example, even though a proton has $\mathrm{MeV}$-order energy around the Bragg peak in water, it can produce a secondary electron with about $2 \mathrm{keV}(\approx 1 \mathrm{MeV} / 460)$ at maximum. Similarly to this, diagnostic X-rays with energies up to several hundreds of keV 
yield quite a few low energy electrons below $10 \mathrm{keV}$ by Compton scattering. The simulation of inelastic processes of these electrons must be an important measure for estimating the production of radical species, e.g., $\mathrm{OH}$ radicals and aqueous electrons, which may cause "indirect effects" of radiation in liquid water.

Figure 2 shows the energy distributions of electrons just before the ionization and electronic excitation collisions per track history for the incident electron energy from $1 \mathrm{keV}$ to $10 \mathrm{keV}$. The incident electron starts with each initial energy and slows down through the energy loss collisions, i.e., ionization and excitation collisions, to rest. As is shown in Fig.2, the energy distributions exhibit specific patterns for the ionization and excitation processes, where the excitation collisions occur with electron energies mostly below $50 \mathrm{eV}$ while the ionization collisions are dominated by high energy electrons around the initial energy and low energy electrons below a few hundred $\mathrm{eV}$. This fact demonstrates the importance of low energy electrons leading to the biological damages presented by Huels et al (2003) [18].

A visualized picture of electron track history in 3D space is shown in Fig.3. Twenty electrons were incident on a water phantom at zero point toward the right direction with initial energy of $3 \mathrm{keV}$. In the picture, red points stand for ionization events and yellow for electronic excitation events. It is shown in the figure that the red and yellow points look rather uniformly distributed. Figures 4(a) and 4(b) show the distributions of flight distance between the successive inelastic collision events. In Fig.4(a), the red curves represent the flight distance distributions between the two ionization events. The blue curves represent the distributions between the two electronic excitation events. In Fig.4(b), the curves in black for the distance distributions between the ionization and the excitation just behind the ionization are also plotted. The reason for the spatial uniformity of the ionization and excitation events seen in Fig. 3 is attributable to the energy distribution of secondary electrons given by the formulation of Eq.(5). According to Eq.(5), the energy partition ratio for the primary and secondary electrons is not even. The energy after the ionization is unequally shared, giving a larger 
portion of the energy to the primary electron. This tendency is more significant as the incident electron energy is larger. Therefore, low energy electrons are inclined to come into being as secondary electrons after the ionization, which leads to the excitation collision events with a high probability. The black curves in Fig.4(b) indicate that the excitation occurs frequently just after the ionization in shorter path length than the other cases (i.e., the distance between the two ionization events or the two excitation events).

Figure 5 shows the event numbers per track history for the ionization and the electronic excitation as a function of the initial electron energy. For example, it can be seen that the incident electron with $1 \mathrm{keV}$-initial energy induces about 40-ionization and 16-excitation events on average per track history. In our simulation, the vibrational excitation and attachment cross sections overlaying the ionization and electronic excitation cross sections around their thresholds have been considered. It was found that the vibrational excitation cross sections affect the event number of the electronic excitation below $30 \mathrm{eV}$. The event counts of the excitation per history rapidly decrease below $30 \mathrm{eV}$ owing to the energy transfer to the vibrational excitation as shown in Fig.5, while the attachment processes have negligible effects on both event numbers. On the other hand, it is a remarkable feature that the rate of the ionization and excitation counts is unique above about $200 \mathrm{eV}$ initial energy. The ratio is roughly 10:4 for the ionization and excitation counts, independently of the initial electron energy. This information should be useful for estimating the excitation event number from a measurement of ionization signal in the experiment and vice versa.

Although the present simulation study was based upon some assumptions of the cross section data for liquid water, the qualitative characteristics as in energy distribution before the inelastic collisions and the spatial uniformity of their event locations have been confirmed to hold even for the change of electronic excitation cross section by $\pm 50 \%$. However, the quantitative evaluations for the ionization and excitation event counts mentioned above are influenced simply by the cross section values; and therefore further investigations are 
necessary from the viewpoint of error propagation arising from the basic data.

\section{Concluding remarks}

In this study, we have carried out Monte Carlo simulations of electrons in liquid water in order to investigate the ionization and excitation collision processes. The simulation results featuring the electron processes are summarized below.

(1) Collision frequencies for inelastic processes (i.e., excitation and ionization) per one track history are distributed in a specific pattern for a given energy range. For example, electronic excitation collisions occur with $10-50 \mathrm{eV}$ electron energy. This is true independent of the initial energy above $\mathrm{keV}$ order of incident electrons, and the count ratio of total events for ionization and excitation processes per history is roughly 10:4 when using the set of cross sections constructed in this study. However, it should be noted that the vibrational excitation processes affect on the frequency of electronic excitation below about $30 \mathrm{eV}$.

(2) The excitation collision is inclined to take place just after the ionization event, which may be attributable to the fact that kinetic energy to primary and secondary electrons after the ionization event is unequally imparted where a large portion of energy is given to the primary electron.

(3) The flight length between inelastic collision events is below $2-3 \mathrm{~nm}$ in liquid water. This is observed with a high probability $(>90 \%)$ of free flights. In addition, in case of successive events with ionization and excitation, the length distribution is shifted to be shorter owing to the tendency in (2).

\section{Acknowledgment}

This work was financially supported in part by the Japan Science and Technology Agency. The authors are indebted to T. Hayashi in the evaluation of the cross section data. 


\section{References}

[1] Olivero J J, Stagat R W, Green A E S (1972) Electron Deposition in Water Vapor, with Atmospheric Applications. J Geophys Res 77(25): 4797-4811

[2] Yousfi M and Benabdessadok (1996) Boltzmann equation analysys of electron-molecule collision cross sections in water vapor and ammonia. J Appl Phys 80(12): 6619-6630

[3] Emfietzoglou D (2003) Inelastic cross-sections for electron transport in liquid water: a comparison of dielectric models. Radiat Phys Chem 66: 373-385

[4] Emfietzoglou D and Nikjoo H (2005) The Effect of Model Approximations on Single-Collision Distributions of Low-Energy Electrons in Liquid Water. Radiat Res 163: 98-111

[5] Emfietzoglou D, Cucinotta F A, Nikjoo H (2005) A Complete Dielectric Response Model for Liquid Water: A Solution of the Bethe Ridge Problem. Radiat Res 164: 202-211

[6] Dingfelder M, Hantke D, Inokuti M, Paretzke H G (1998) Electron inelastic-scattering cross sections in liquid water. Radiat Phys and Chem 53: 1-18

[7] Pimblott S M and Siebbeles L D A (2002) Energy loss by non-relativistic electrons and positrons in liquid water. Nucl Instr and Meth B194: 237-250

[8] Champion C (2003) Theoretical cross sections for electron collisions in water: structure of electron tracks. Phys Med Biol 48: 2147-2168

[9] Seng G and Linder F (1976) Vibrational excitation of polar molecules by electron impact. II. Direct and resonant excitation in $\mathrm{H}_{2} \mathrm{O}$. J Phys B: At Mol Phys 7: 2539-2551

[10] El-Zein A A A, Brunger M J, Newell W R (2000) Excitation of vibrational quanta in water by electron impact. J Phys B: At Mol Opt Phys 33: 5033-5044

[11] Märk T D, Hatano Y, Linder F (1995) IAEA-TECDOC 799 "3. Electron Collision Cross Sections": 163-275

[12] Melton C E (1972) Cross Sections and Interpretation of Dissociative Attachment Reactions Producing $\mathrm{OH}^{-}, \mathrm{O}^{-}$, and $\mathrm{H}^{-}$in $\mathrm{H}_{2} \mathrm{O}^{*}$. J Chem Phys 57: 4218-4225

[13] Danjo A and Nishimura $\mathrm{H}$ (1985) Elastic Scattering of Electrons from $\mathrm{H}_{2} \mathrm{O}$ Molecule. J Phys Soc Japan 54: 1224-1227

[14] Itikawa Y and Mason N (2005) Cross Sections for Electron Collisions with Water Molecules. J Phys Chem Ref Data 34(1): 1-22

[15] Brenner D J and Zaider M (1983) A computationally convenient parameterization of experimental angular distributions of low energy electrons elastically scattered off water 
vapour. Phys Med Biol 29(4): 443-447

[16] Grosswendt B and Waibel E (1978) Transport of low energy electrons in nitrogen and air. Nucl Instr and Meth 155: 145-156

[17] Green A E S and Sawada T (1972) Ionization cross sections and secondary electron distributions. J Atmosph Terr Phys 34: 1719-1728

[18] Huels M A, Boudaïffa B, Cloutier P, Hunting D, Sanche L (2003) Single, Double, and Multiple Double Strand Breaks Induced in DNA by 3-100eV Electrons. J Am Chem Soc 125: 4467-4477 


\section{Figure captions}

\section{Figure 1.}

A set of electron collision cross sections used in the present study. The elastic collision cross section $\mathrm{q}_{\mathrm{els}}$ is taken from Danjo and Nishimura (1985) [13] with a multiplication factor of 1.4, and it is set to be constant below the cutoff energy of simulation $(8.22 \mathrm{eV})$. The total ionization cross section $\mathrm{q}_{\mathrm{ion} \mathrm{T}}$ and the total electronic excitation cross section $\mathrm{q}_{\mathrm{excT}}$ are taken from Emfietzoglou (2003) [3], and the attachment cross sections are from Melton (1972) [12]. For the vibration excitation cross sections $\mathrm{q}_{\mathrm{vib}}$, three lump modes, $(100+001),(010)$ and others, are considered. The cross sections for $(100+001)$ and (010) are estimated by a combination of the data from Seng and Linder (1976) [9] and from El-Zein et al (2000) [10]. The summed cross section for the other modes is constructed referring to Märk et al (1995) [11].

\section{Figure 2.}

Electron energy distributions just before the excitation and ionization collisions per track history. The incident electron is assumed to start with initial energies, 1, 2, 3, 5, 7 and 10keV.

\section{Figure 3.}

Visualized picture of electron track history in 3D space. Twenty electrons are incident on a water phantom at zero point toward the right direction. Initial energy of the electrons is $3 \mathrm{keV}$. Red points stand for ionization events and yellow for excitation events.

\section{Figure 4.}

(a) Flight length distributions between excitation events and between ionization events.

(b) Flight length distributions between ionization and successive excitation events. It is shown that the excitation event is inclined to occur just after the ionization event in a short interval.

\section{Figure 5.}

Total counts for excitation and ionization events per track history as a function of the initial energy of incident electron. 


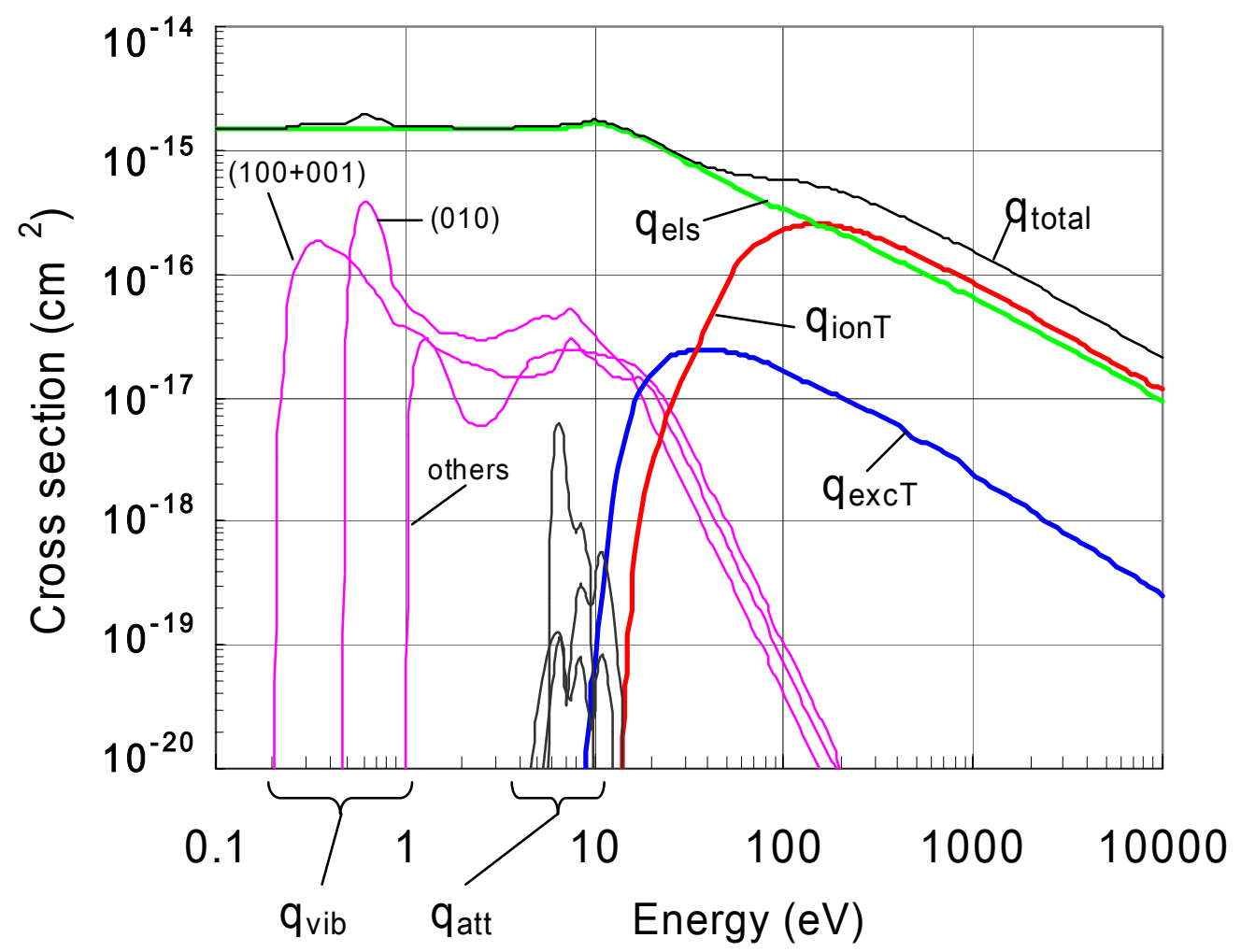

Figure 1.

Date et al. 


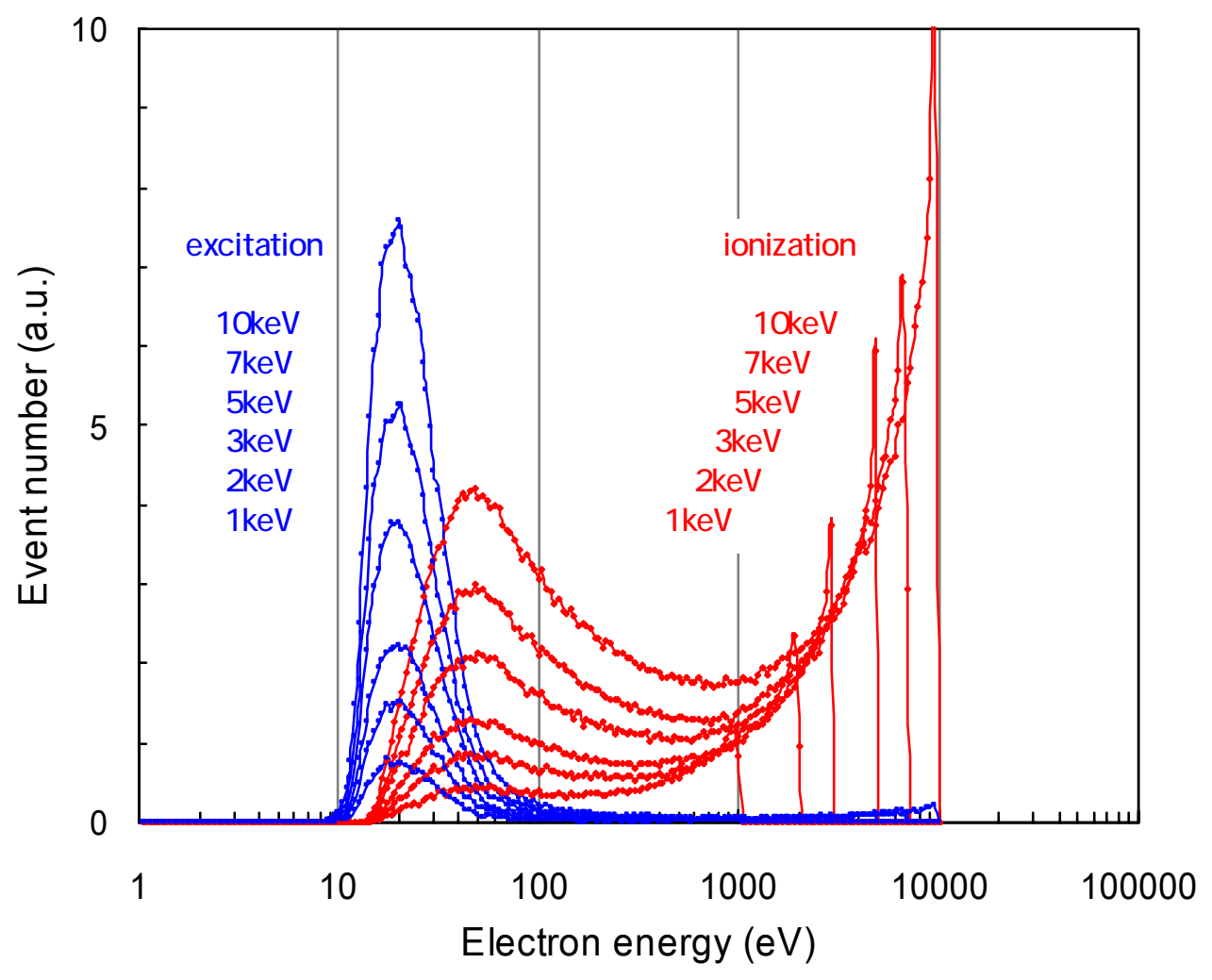

Figure 2.

Date et al. 


\section{Electron Trajectory}

ionization event

- excitation event

100nm)

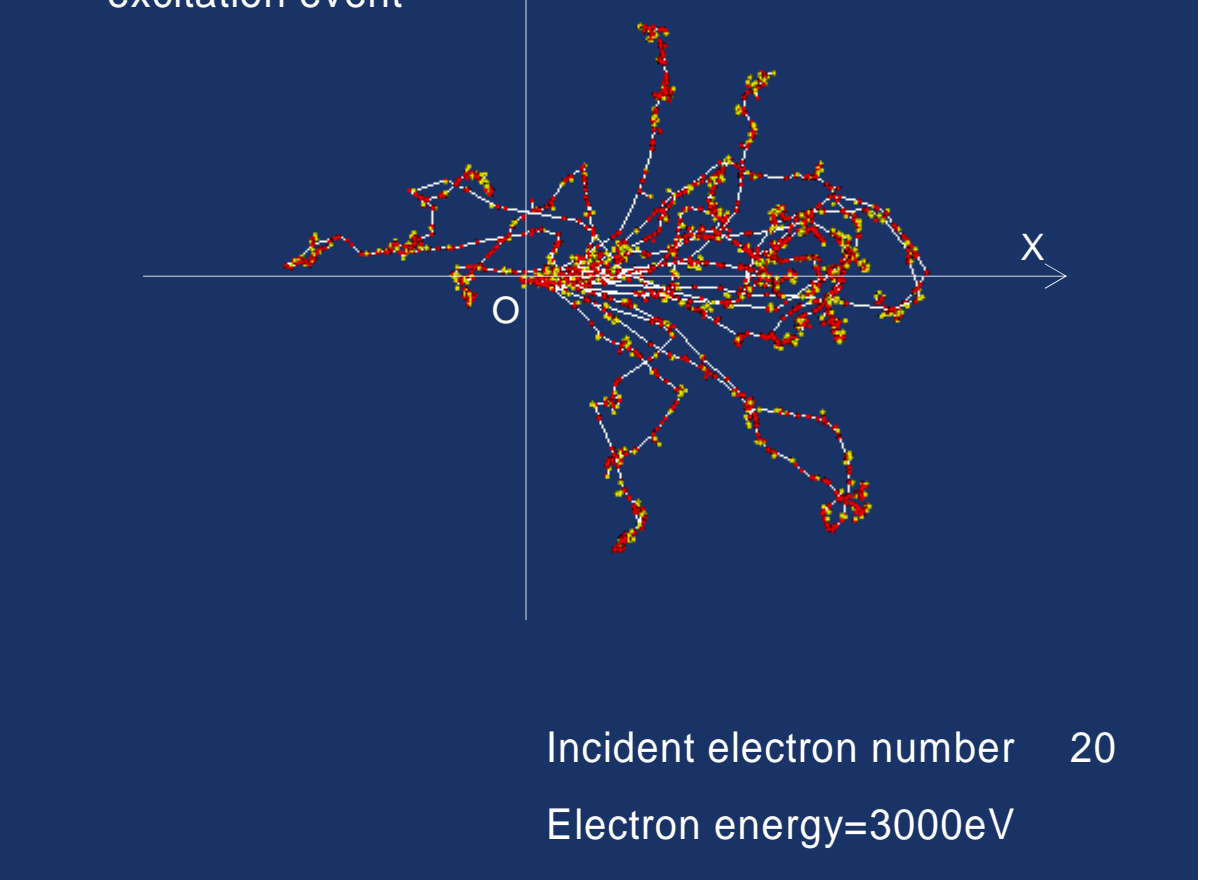

Figure 3.

Date et al . 


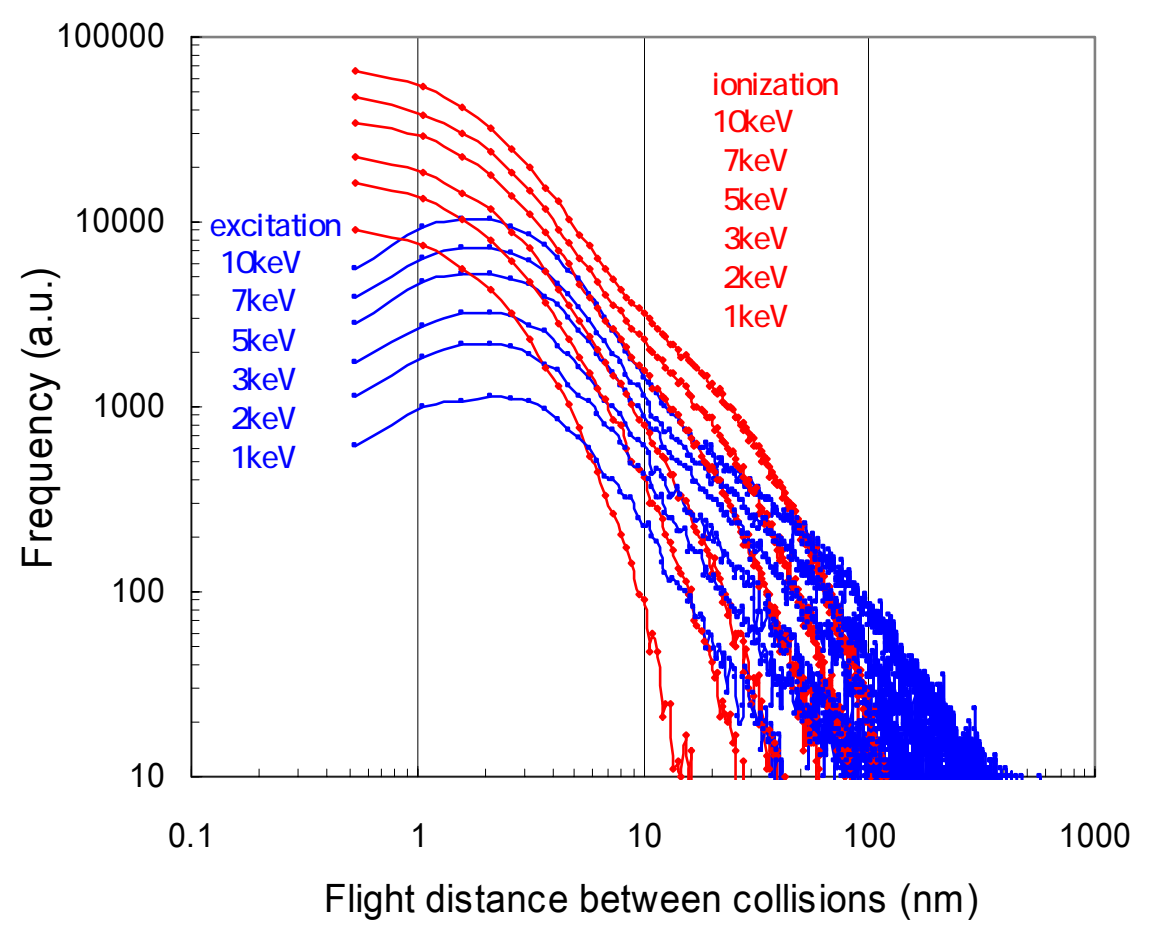

(a)

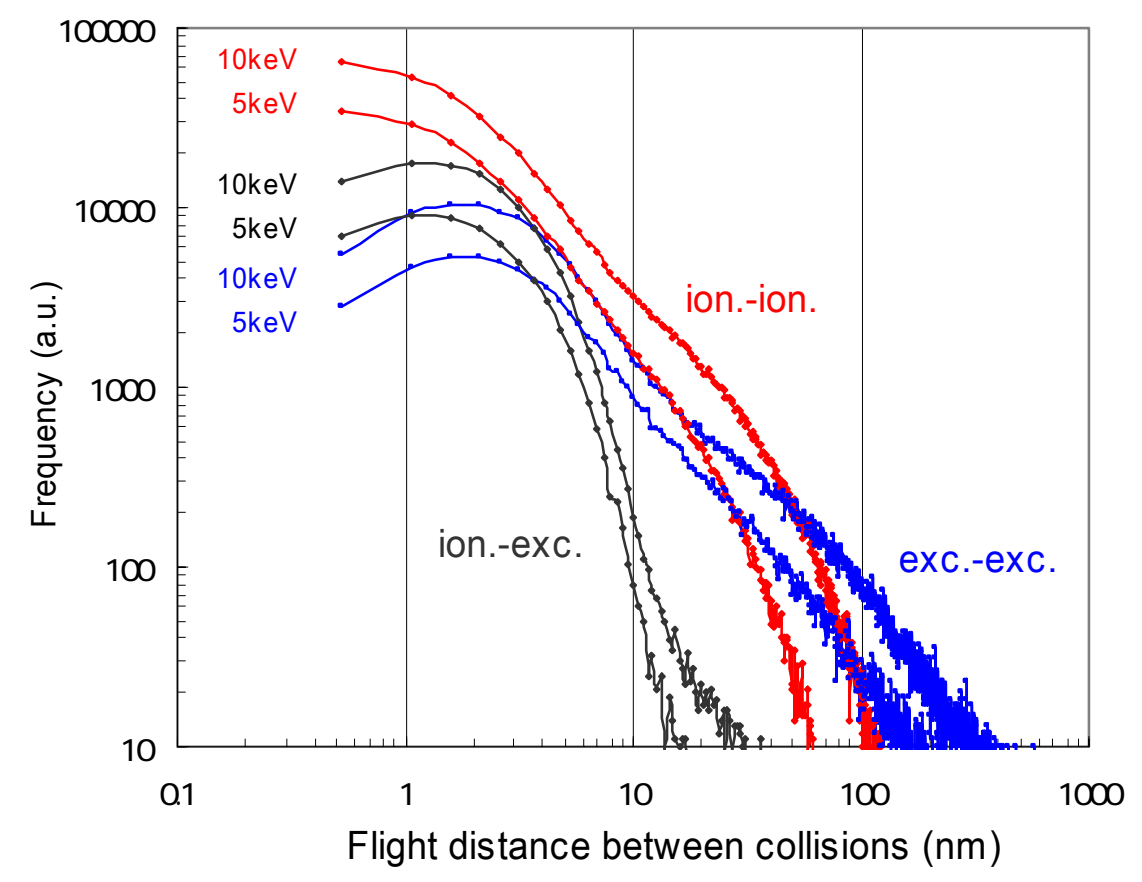

Figure 4. (a), (b)

Date et al. 


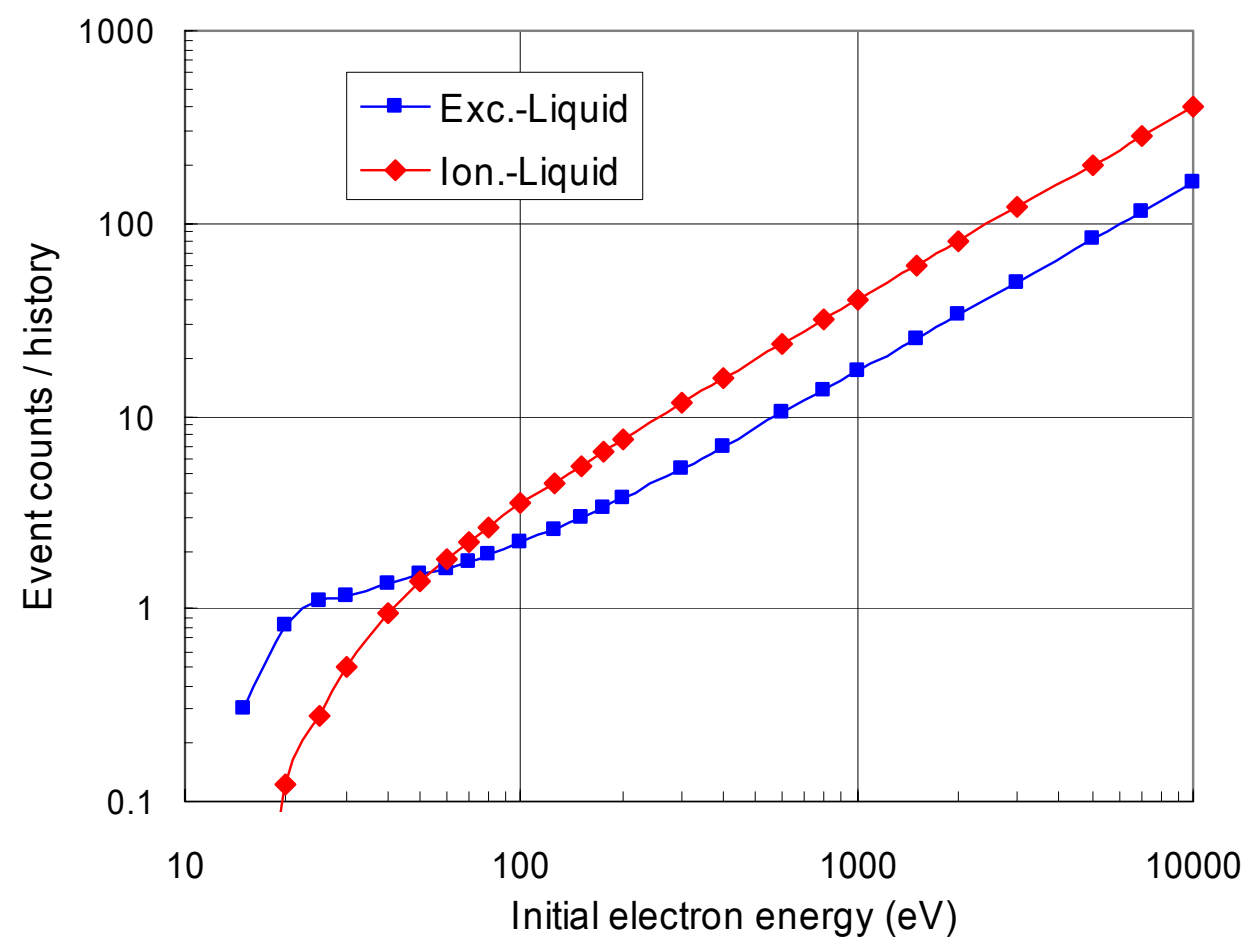

Figure 5.

Date et al . 